\section{Taphonomy: Definition}

Soren Blau

Victorian Institute of Forensic Medicine /

Department of Forensic Medicine, Monash

University, Melbourne, VIC, Australia

The term taphonomy (from the Greek taphos- $\tau$ á law) is defined as the study of the transition of plant and animal organisms after death from the biosphere (living surfaces) to the lithosphere (underground). Taphonomists study processes such as decomposition or burial that affect organisms after death and which ultimately result in animals and plants becoming part of the fossil record.

\section{Cross-References}

Taphonomy/Time Since Death 